\title{
Pure Lumbar Epidural Cavernous Hemangioma with Contralateral Symptoms Complicated by Spondylolisthesis
}

\author{
Pan Qiao1, Tiantong Xu1, Wen Zhang2, Wenyuan Ding3 and Rong Tian1 \\ ${ }^{1}$ Department of Spine Surgery, Tianjin Union Medical Center, Tianjin, China \\ ${ }^{2}$ Department of Pneumology, Tianjin Children's Hospital, Tianjin, China \\ ${ }^{3}$ Department of Spine Surgery, The Third Hospital of Hebei Medical University, China
}

\begin{abstract}
Pure spinal epidural cavernous hemangioma with contralateral symptoms is rarely reported. It will be very difficult to diagnose when it is complicated by contralateral symptoms. A 56-year female presented with pure lumbar epidural cavernous hemangioma with contralateral symptoms. Physical examination and imaging including X-ray, CT and MRI, suggested that it is a hemangioma complicated by spondylolisthesis. Laminectomy and exploration was performed. Postoperative X-ray showed satisfactory interbody fusion and reduction of spondylolisthesis. The patient was fully recovered four months postoperatively. Histopathological examination showed that the mass was epidural cavernous hemangioma.
\end{abstract}

Key Words: Cavernous hemangioma, Spondylolisthesis, Hemilaminectomy.

How to cite this article: Qiao P, Xu T, Zhang W, Ding W, Tian R. Pure lumbar epidural cavernous hemangioma with contralateral symptoms complicated by spondylolisthesis. J Coll Physicians Surg Pak 2019; 29(10):1003-5.

\section{INTRODUCTION}

Pure spinal epidural cavernous hemangioma is a benign vascular malformation, accounting for $0.04-1.2 \%$ of all spinal axis tumors. It is commonly observed within a vertebral body, extending into the epidural space. ${ }^{1}$ Pure spinal epidural cavernous hemangiomas without bony involvement are particularly uncommon, accounting for $4 \%$ of all spinal epidural tumors. ${ }^{2}$ These are usually observed in the lumbar and thoracic spine rather than in the cervical spine. The spinal cavernous hemangiomas with contralateral symptom are not commonly seen, which make them difficult to diagnose.

We hereby present a case of a pure lumbar epidural cavernous hemangioma complicated by contralateral symptoms that were similar to sequestrated lumbar disc herniation. The patient was successfully treated with hemilaminectomy and interbody fusion.

\section{CASE REPORT}

A 56-year female was suffering from low back pain, after walking for 10 years; and numbness in posterior aspect of left leg and left foot sole for 1 year. She went to a local hospital and was treated with conservative therapies including anti-inflammatory drugs and traditional Chinese

Correspondence to: Dr. Rong Tian, Department of Spine Surgery, Tianjin Union Medical Center, No.190, Jieyuan Road,

Hongqiao District, Tianjin, 300121, China

E-mail: tianrong067@163.com

Received: October 23, 2018; Revised: March 17, 2019; Accepted: March 28, 2019 acupuncture and massage for 6 weeks. But those symptoms persisted.

She was admitted to the hospital in April, 2016. Physical examination found that there was hypalgesia in the posterior aspect of the left leg and move severed hypalgesis in the left foot sole. X-ray examination revealed Grade I intact arch spondylolisthesis of $L 4$ vertebrae (Figure 1A), with reduced bilateral Achilles tendon reflexes and normal muscle strength. Neurologic examination revealed S1 dermatomal hypoesthesia in the left side. CT scan showed a mass, with similar density to the intervertebral disc, in the right anterior epidural area of L5/S1 disc (Figures 1B-C). MRI showed that the mass was heterogonous isointense on $\mathrm{T} 1$, which showed mixed intensity on T2-weighted images (Figures 1D, E). It was diagnosed as hemangioma complicated by lumbar spondylolisthesis.

Considering the above diagnosis, a bilateral hemilaminectomy with left medial facetectomy of L4 and a complete laminectomy of L5 were performed; in addition, interbody fusion of L4/L5 was performed with a cage. Intraoperatively, it was observed that grade-I intact arch spondylolisthesis in L4 vertebrae and step sign at posterior aspect of L4-5 disc. There was no disc herniation at L5-S1 or ruptured annulus fibrosis. A dark reddish, spherical and well-circumscribed lesion was observed in the right anterior epidural area at L5/S1 disc. The lesion was completely removed. The construct was augmented with pedicle screw fixation at L4-S1.

The postoperative course was uneventful and X-ray showed normal height of intervertebral space and reduction of 
spondylolisthesis two-weeks postoperatively (Figure 2). After 4-month follow-up, the patient showed no symptoms and was fully recovered. Histopathological examination showed that the mass was a $13 \times 8 \times 2 \mathrm{~mm}$ cavernous haemangioma that was composed of single-layer-lined

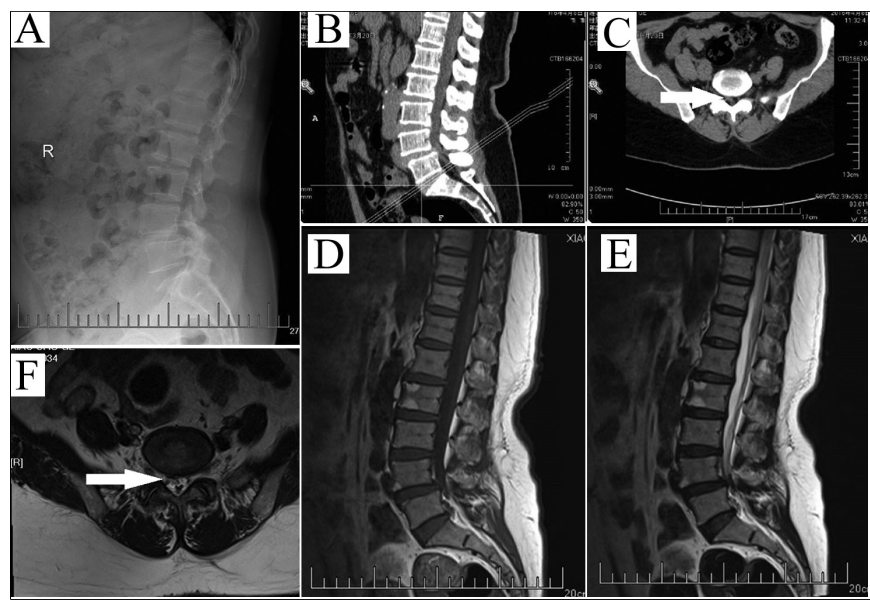

Figure 1: Preoperative imaging of the mass. (A) X-ray demonstrating grade I intact arch spondylolisthesis of L4 vertebrae. (B, C) CT scan showing a mass, with similar density to the intervertebral disc, in the right anterior epidural area of L5/S1 disc. (D, E) Sagittal MR-imaging showing heterogonous isointense on T1- (D) and hypo- and hyperintense on T2-weighted images $(E)$.

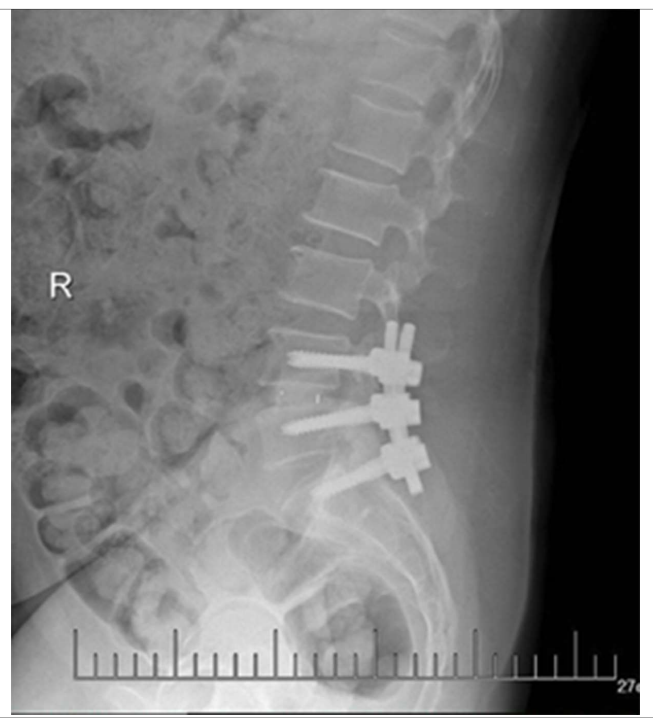

Figure 2: Postoperative X-ray showing satisfactory interbody fusion of L4/L5, pedicle screw fixation of L4-S1, and reduction of spondylolisthesis.

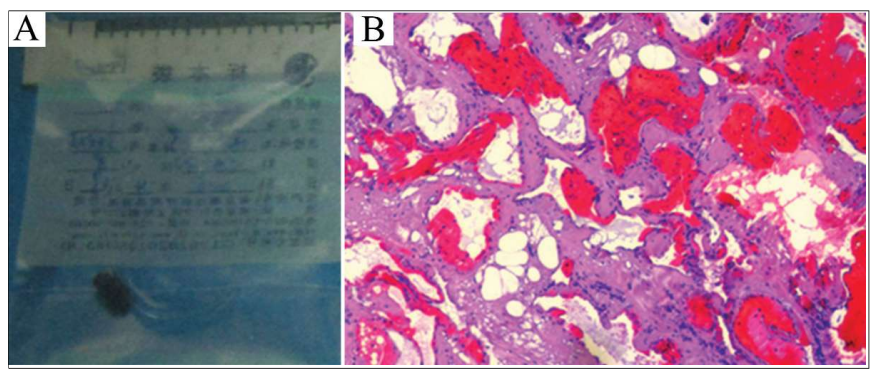

Figure 3: Postoperative histopathological examination. (A) The mass was dark reddish, spherical, well circumscribed and $13 \times 8 \times 2 \mathrm{~mm}$ in size. (B) The lesion was mainly composed of single-cell layer-lined vascular structures and connective tissue (HE staining, $\mathrm{x} 40$ ) vascular channels and connective tissue (Figures 3A, B). No evidence of malignancy was found.

\section{DISCUSSION}

There are very few reports on the pure spinal epidural cavernous hemangiomas with contralateral symptoms and complicated by spondylolisthesis. The development process of epidural hemangiomas is usually slow and may result in compression of the spinal cord. The clinical manifestations vary with the compression site. Therefore, it would be difficult to make a diagnosis when it is complicated by contralateral symptoms. In this patient, CT scan revealed mild bony and ligamentous stenosis on the S1 nerve root canal at the left side. There were no prominent fat, venous congestion or disc herniation in the spinal canal except for the mass. The traction on the left S1 nerve root was slightly greater. We then performed other examinations, which suggested that the contralateral symptoms were the result of traction force, just as contralateral symptoms result from disc herniation.

Differential diagnosis of epidural cavernous hemangiomas includes disc herniation, meningiomas, and malignant tumors among others. In this case, it was firstly suspected the mass to be a sequestrated disc. However, disc herniation usually occurs in the ventral part and presents unchanged signal without enhancement on MR images. MRI T2-weighted image signals of the mass were inconsistent with the signals of L4/5 disc. The signals of meningiomas are usually isointense on T1and T2-weighted images, and homogeneously enhance with characteristic sign of dura tail after contrast administration. ${ }^{3}$ Epidural malignant tumors generally present an aggressive clinical course with adjacent vertebral bony involvement. Angiolipoma is typically hyperintense on T1-weighted images, ${ }^{4}$ while fat is commonly absent in cavernous hemangiomas and if present, it is peripherally located. Therefore, MRI, especially enhanced MRI, is essential to avoid misdiagnosis.

Complete surgical resection is the treatment of choice for epidural cavernous hemangiomas. Because of excessive vascularity, en bloc removal of the tumor is recommended, which can reduce blood loss, resulting from piece-by-piece resection. Bipolar coagulation in the superficial and surrounding part of the mass is also recommended. ${ }^{5}$ Radiotherapy is not generally used for epidural cavernous hemangiomas due to the radiation damage to the spinal cord. As for the surgical approaches for lumbar disc herniation with contralateral symptoms, both unilateral exploration on the herniated side and bilateral exploration have been reported. However, neither of them have been used to treat lumbar epidural cavernous hemangiomas complicated by contralateral symptoms. In this patient, significant symptom remission and satisfactory neural decom-pressions were obtained via exploration on both sides. 
In conclusion, the surgery was effective in this case and the patient recovered well. The authors suggest that it is critical to use appropriate imaging for differential diagnosis of pure spinal epidural cavernous hemangiomas complicated by contralateral symptoms to avoid misdiagnosis and mismanagement.

\section{FUNDING:}

This study was funded by the Research Fund of Tianjin Union Medical Center (2017YJ025).

\section{PATIENTS' CONSENT:}

Informed consent is obtained from patient to publish the data concerning this case.

\section{CONFLICT OF INTEREST:}

Authors declared no conflict of interest.

\section{AUTHORS' CONTRIBUTION:}

$P Q$ : Study design, data collection, result interpretation and manuscript draft.
TX: Data collection, result interpretation and manuscript draft.

WZ: Data collection, study design and manuscript draft. WD: Data collection, result analysis and manuscript draft. RT: Study design, manuscript revision and manuscript submission.

\section{REFERENCES}

1. Andaluz N, Balko G, Bui H, Zuccarello M. Angiolipomas of the central nervous system. J Neurooncol 2000; 49:219-30.

2. Nagi S, Megdiche H, Bouzaïdi K, Haouet S, Khouja N, Douira W, et al. Imaging features of spinal epidural cavernous malformations. J Neuroradiol 2004; 31:208-13.

3. Zhong W, Chen H, Huang S, You C. Cervical en-plaque epidural meningioma. Neurol India 2011; 59:132-4.

4. Gelabertgonzález M, Garcíaallut A. Spinal extradural angiolipoma: Report of two cases and review of the literature. Eur Spine J 2009; 18:324-35.

5. Talacchi A, Spinnato S, Alessandrini F, luzzolino P, Bricolo A. Radiologic and surgical aspects of pure spinal epidural cavernous angiomas. Report on 5 cases and review of the literature. Surg Neurol 1999; 52:198-203. 